\title{
A Simple Game to Introduce Scrum Concepts
}

\author{
Stan Kurkovsky \\ Central Connecticut State University \\ New Britain, CT, USA \\ kurkovsky@ccsu.edu
}

\begin{abstract}
It is often very difficult to transition from the waterfall to an agile process. To simplify this transition, we designed a LEGO game to introduce basic Scrum concepts and principles. This game provides students with an opportunity to play different scrum roles and measure the development team velocity in two sprints.
\end{abstract}

\section{CCS CONCEPTS}

- Software and its engineering $\rightarrow$ Agile software development; Waterfall model;

\section{KEYWORDS}

Software process, agile, scrum, sprint, game, LEGO

\section{ACM Reference Format:}

Stan Kurkovsky. 2020. A Simple Game to Introduce Scrum Concepts. In The 51st ACM Technical Symposium on Computer Science Education (SIGCSE '20), March 11-14, 2020, Portland, OR, USA. ACM, New York, NY, USA, 1 page. https://doi.org/10.1145/3328778.3372593

\section{BACKGROUND}

Although the waterfall model works well for instructional purposes, it is often difficult for a novice software developer to transition from a plan-based to an iterative process. Based on the previous work of using LEGO to teach sofware engineerin (e.g. [1]), we present a 30 -minute game ${ }^{1}$ aimed to introduce iterative process principles along with Scrum concepts. A number of Scrum games are currently in use by agile coaches and educators (e.g. lego4scrum.com). However, they are often difficult to fit into a single class period due to time requirements, they focus specifically on Scrum rather than on broader iterative development principles, and they do not offer a direct comparison of plan-driven and iterative processes.

\section{METHODS}

Our Tetris-based game requires basic familiarity with the concept of software process. It is played by several pairs of teams, Team A and Team B. An ideal size of each team is 2-3 people, but there's no limit on the number of teams. Each pair of teams needs a small LEGO baseplate of the same size, which will serve as the Tetris well, and a selection of standard LEGO bricks. During the game,

${ }^{1}$ https://web.ccsu.edu/lego-se/modules/IntroToScrum.html

Permission to make digital or hard copies of part or all of this work for personal or classroom use is granted without fee provided that copies are not made or distributed for profit or commercial advantage and that copies bear this notice and the full citation on the first page. Copyrights for third-party components of this work must be honored.

For all other uses, contact the owner/author(s).

SIGCSE '20, March 11-14, 2020, Portland, OR, USA

(C) 2020 Copyright held by the owner/author(s).

ACM ISBN 978-1-4503-6793-6/20/03.

https://doi.org/10.1145/3328778.3372593 each brick needs to be placed on the baseplate as if it fell down the well: either at the bottom, or immediately above another brick. No bricks can be placed below another brick. Unlike the classical game, completed rows of bricks do not disappear. The game is over when a brick cannot be placed completely on the baseplate.

The game is played in two rounds. Round 1 simulates a plandriven process. Each Team A makes a "plan" by arranging all bricks into a queue, in which no bricks of the same size can be adjacent to each other. Then, Team A takes one brick at a time from the queue and gives it to Team B, which places the brick on the baseplate following the rules. They continue until no more bricks can fit entirely on the baseplate. Each Team B is asked to calculate their quality metric as the number of baseplate studs covered by bricks divided by the size of the entire baseplate.

Round 2 simulates an iterative process. Teams A and B switch roles: Team $B$ has the bricks in the product backlog, while Team A puts them in the well. The rules are the same, but instead of handing out one brick at a time, Team $\mathrm{B}$ gives five bricks from the top of the queue to Team A. These five bricks simulate a sprint backlog. Team A decides in which sequence to place these bricks on the baseplate following the rules described above. As such, Team A acts as a Scrum development team that determines how to do the work, while Team B acts as a product owner who decides what work needs to be done. Team A repeats the previous step until the game is over and no more bricks can fit entirely on the baseboard. Otherwise, Team A starts the next sprint by asking Team B for five more bricks. At the end of the game, each Team A calculates their quality metric, which mimics team velocity representing the amount of work accomplished by the development team.

A debrief concludes the game by comparing average quality metrics, which are typically higher for Round 2. Students are asked to suggest the reasons for this difference. It is important to mention to students that plan-driven development does not give the teams much freedom to anticipate change, which may result in lack of efficiency. Students should also be asked how they felt about Round 2 of the game. They frequently mention that the rules of Round 2 allowed them to better see the bigger picture and plan their work within the iteration yielding better results. This is usually a good moment to introduce the similarities between Scrum and Round 2 of the game identifying key Scrum concepts highlighted above.

\section{ACKNOWLEDGMENTS}

This work is supported in part by the National Science Foundation IUSE award 1611905.

\section{REFERENCES}

[1] Stan Kurkovsky, Stephanie Ludi, and Linda Clark. 2019. Active Learning with LEGO for Software Requirements. In Proceedings of the 50th ACM Technical Symposium on Computer Science Education (SIGCSE '19). ACM, New York, NY, USA, 218-224. https://doi.org/10.1145/3287324.3287444 